\title{
Checklists in Neurosurgery to Decrease Preventable Medical Errors: A Review
}

\author{
Yavor Enchev \\ Department of Neurosurgery, Medical University of Varna, St. Marina University Hospital, Varna, Bulgaria
}

Neurosurgery represents a zero tolerance environment for medical errors, especially preventable ones like all types of wrong site surgery, complications due to the incorrect positioning of patients for neurosurgical interventions and complications due to failure of the devices required for the specific procedure.

Following the excellent and encouraging results of the safety checklists in intensive care medicine and in other surgical areas, the checklist was naturally introduced in neurosurgery. To date, the reported world experience with neurosurgical checklists is limited to 15 series with fewer than 20,000 cases in various neurosurgical areas.

The purpose of this review was to study the reported neurosurgical checklists according to the following parameters: year of publication; country of origin; area of neurosurgery; type of neurosurgical procedureelective or emergency; person in charge of the checklist completion; participants involved in completion; whether they prevented incorrect site surgery; whether they prevented complications due to incorrect posi- tioning of the patients for neurosurgical interventions; whether they prevented complications due to failure of the devices required for the specific procedure; their specific aims; educational preparation and training; the time needed for checklist completion; study duration and phases; number of cases included; barriers to implementation; efforts to implementation; team appreciation; and safety outcomes.

Based on this analysis, it could be concluded that neurosurgical checklists represent an efficient, reliable, cost-effective and time-saving tool for increasing patient safety and elevating the neurosurgeons' self-confidence. Every neurosurgical department must develop its own neurosurgical checklist or adopt and modify an existing one according to its specific features and needs in an attempt to establish or develop its safety culture. The world, continental, regional and national neurosurgical societies could promote safety checklists and their benefits.

Keywords: Checklist, neurosurgery, patient safety, medical errors
Neurosurgery, although being one of the most high-tech surgical areas, is not reliably protected against preventable medical errors like all subtypes of incorrect surgery site, complications due to incorrect positioning of the patients for neurosurgical interventions and complications due to failure of the devices required for the specific procedure. In an attempt to increase patient safety and reduce the risk factors, check- lists were introduced and implemented in this zero tolerance environment. Neurosurgical checklists represent both source and practical expression of a safety culture in neurosurgery.

The primary objective of this review was to highlight the current state of the checklist application in neurosurgery as well as to outline its future trends concerning a reduction of preventable medical errors. 


\section{PREVENTABLE MEDICAL ERRORS IN NEUROSURGERY}

Medical errors can be defined as avoidable adverse effects of medical care, regardless of whether or not they are obvious or damaging for the patient (1).

The preventable medical errors in neurosurgery, which are the objective of this review, include all subtypes of incorrect surgery site, complications due to incorrect positioning of the patients for neurosurgical interventions and complications due to a failure of the devices required for the specific procedure.

\section{Incorrect site surgery}

The term "wrong site surgery" is often used as a general designation of several subtypes of incorrect site surgical events: incorrect site surgery (in particular) - surgical procedure accomplished on an incorrect body part; wrong level surgery - surgical procedure performed at an erroneous level but at the correct site; wrong level exposure - surgical exposure completed on a mistaken level; wrong side surgery - surgical procedure performed on the wrong side of the body or on the wrong extremity; incorrect procedure - the wrong surgical procedure performed on the right side and site; and incorrect patient - intervention performed on the wrong patient (2).

\section{Complications due to incorrect positioning of the patients for neurosurgical procedures}

Positioning of neurosurgical patients is an important part of every procedure and paying attention to the physical and physiological consequences of incorrect positioning can prevent serious adverse events and complications. Ideal patient positioning involves balancing surgical comfort against the risks related to the patient position.

Complications due to incorrect positioning of the patients for neurosurgical procedures include perioperative nerve injury and postoperative visual loss (3). The perioperative nerve injury could be brachial plexus injury, ulnar neuropathy, median neuropathy and radial neuropathy. The most common causes of postoperative visual loss are ischemic optic neuropathy and central retinal artery occlusion (3).

\section{Complications due to failure of the devices required for the procedure}

Most of the neurosurgical procedures require devices for their completion, like high speed drills, operating microscopes, neuroendoscopes, ultrasounds, $\mathrm{C}$-arms, neuronavigation, cavitron ultrasonic surgical aspirators, etc. In the case of malfunction of such devices, depending on the stage of the intervention, the surgery may not be performed at all, or could fail to be completed in the preoperatively planned manner and extent.

\section{RISK FACTORS FOR PREVENTABLE MEDICAL ERRORS IN NEUROSURGERY}

Neurosurgery represents the third most amenable surgical area to wrong site surgery next to orthopedics and general surgery (4).

Risk factors to wrong site surgery include incorrect patient positioning or preparation of the operative site, erroneous information provided by the patient or their family, missing or improper patient consent, failure to use site markings, neurosurgeon exhaustion, several neurosurgeons involved in one case, multiple procedures on the same patient, unusual time limits, emergent procedures, unusual patient anatomy, and overall poor operative team communication (2).

Positioning of neurosurgical patients is challenging in its complexity and variability. It is the responsibility of both the neurosurgeon and the anesthesiologist. On the one hand, it requires an adequate anesthetic depth, maintenance of hemodynamic stability, evidence of appropriate oxygenation, and the preservation of invasive monitors. On the other hand, there is a necessity of optimal physiological position of the head, eyes, neck, extremities, breasts and genitals, without any abnormal compression, traction, flexion or extension (3).

Most of the neurosurgical operations could not be accomplished without excellent technical support by heterogeneous specialized high-tech equipment. The perfect condition of these indispensable devices required for every neurosurgical procedure is obligatory for its uneventful course.

In an attempt to cope with the aforementioned risk factors for preventable medical errors in neurosurgery, safety checklists were introduced.

\section{CHECKLIST DEFINITION}

The checklist, by definition, represents a tool for collecting information aiming to prevent the failure of some human activity due to the inherent limitations of human attention and memory. It guarantees the sequence and accomplishment of the planned assignment (5).

\section{NEUROSURGICAL CHECKLISTS}

The neurosurgical checklists reported in the literature to date are summarized and juxtaposed in Table 1 and 2.

\section{NEUROSURGICAL CHECKLISTS}

Distribution by year

All but one of the studies reporting neurosurgical checklists were published within the last 6 years. The first safety surgical 


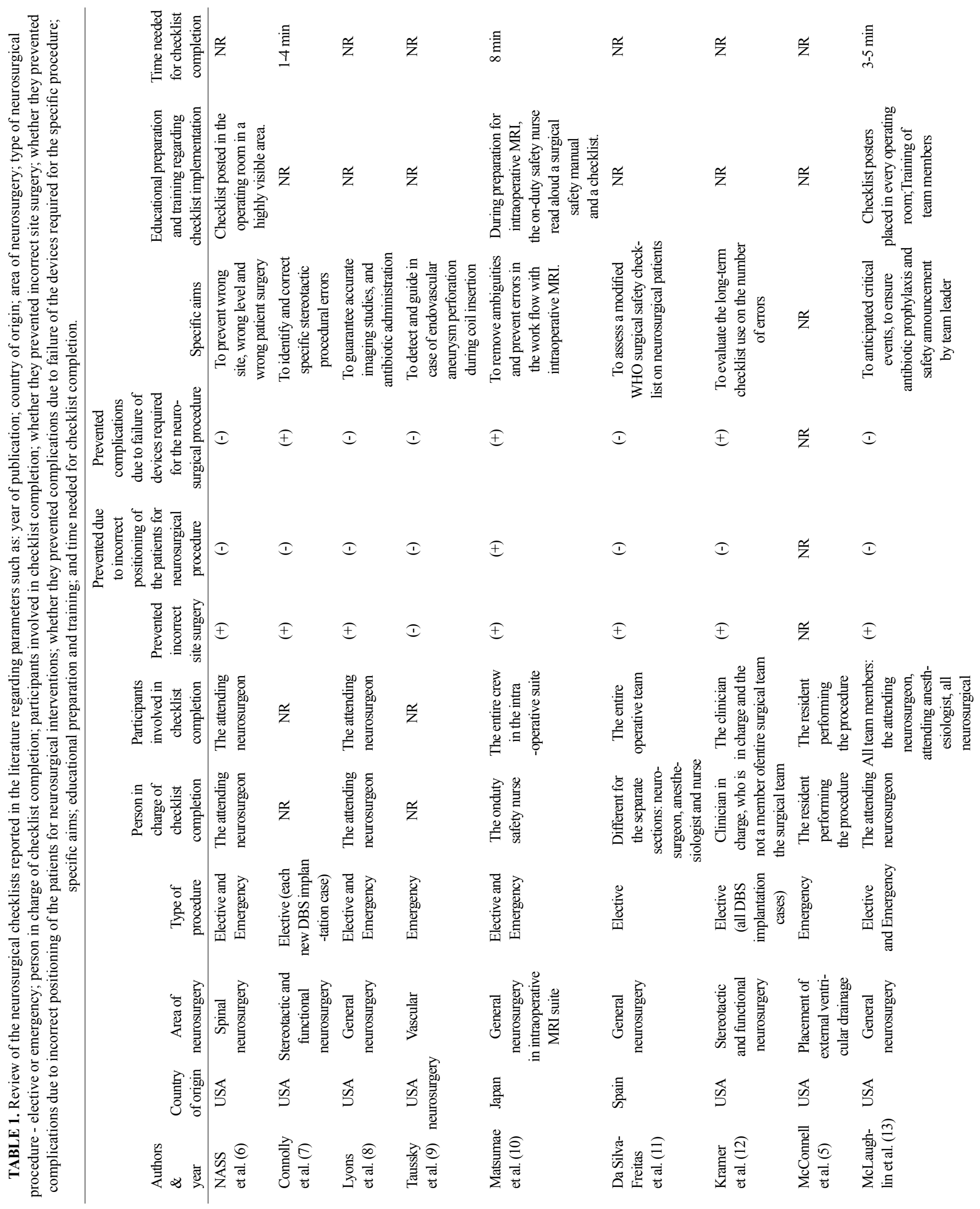




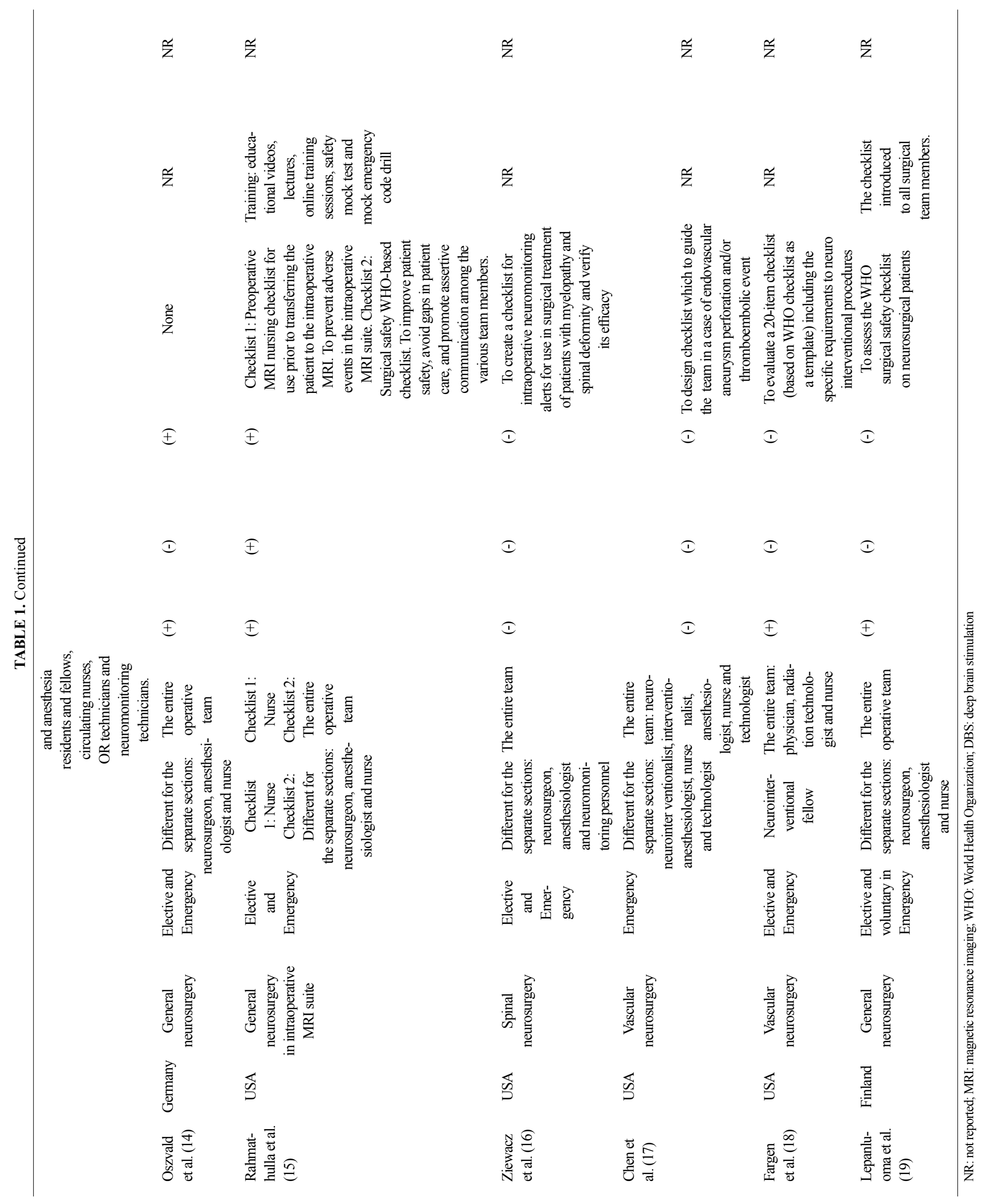


checklist concerning neurosurgery was that of North American Spine Society and was published in 2001 (6). It aimed to prevent incorrect site, incorrect level and wrong patient surgery in spinal and orthopedic cases. The second neurosurgical checklist was in the field of stereotactic and functional neurosurgery and was reported only eight years later when Connolly et al. (7) published their checklist designed especially for elective new deep brain stimulation (DBS) implantation cases. The largest number of papers describing neurosurgical checklists and their results was observed in 2012 (almost half of all reports - 7/15) (5,11-16).

\section{Country of origin}

Overall, 11 out of 15 (69\%) papers reporting neurosurgical checklists were from the USA $(5-9,12,13,15-18)$. Three studies originated from Europe (Spain (11), Germany (14) and Finland (19) and 1 was from Asia (Japan) (10). These data clearly demonstrate that the problem of patient safety in neurosurgery is more real and important for colleagues from the USA, most probably due to the peculiarities of their health system and the traditionally higher level of medicolegal issues there. The increasing popularity and influence of patient organizations, as well as the ever-reducing tolerance in modern society to medical errors, especially preventable ones, will most likely stimulate the wider distribution of neurosurgical safety checklists in Europe.

\section{Area of neurosurgery}

Overall, 7 out of 15 neurosurgical checklists were reasonably designed and applied in general neurosurgery $(8,10,11,13-$ $15,19), 3$ were in vascular neurosurgery $(9,17,18), 2$ in stereotactic and functional neurosurgery $(7,12)$, another 2 were in spinal neurosurgery $(6,16)$ and 1 was regarding the placement of external ventricular drainage (5). Two of the general neurosurgery checklists were developed to guide and prevent errors in an intraoperative magnetic resonance imaging (MRI) suite $(10,15)$. These numbers clearly reflected the case distribution in the common neurosurgical practice.

\section{Type of neurosurgical procedures}

Most of the checklist studies include both elective and emergency cases ( 8 out of 15$)(6,8,10,13-16,18)$. One checklist was applied in elective and voluntarily in emergency procedures (19). Three of the neurosurgical checklists were limited only to elective surgeries $(7,11,12)$ and the same number of checklists were implemented for emergency cases $(5,9,17)$.

\section{Person in charge for the checklist completion}

This characteristic of the neurosurgical checklists was quite variable. The reported experience included several possibilities for the person in charge to complete the checklist. These were different persons (neurosurgeon, anesthesiologist and nurse or technologist) for each particular section of the checklist $(6 / 15)(11,14-17,19)$, the attending neurosurgeon $(3 / 15)$ $(6,8,13)$, the resident performing the procedure $(1 / 15)(5), a$ neurointerventional fellow (1/15) (18), a clinician in charge who is not from the surgical team (1/15) (12), and the on-duty safety nurse (1/15) (10). In 2 studies, the person in charge of the safety checks was not reported $(7,9)$.

\section{Participants in the checklist completion}

In an attempt to enhance team workflow and communication, most of the neurosurgical checklists required the participation of the entire operative team (10/15) (10-19). However, some of them needed only the attending neurosurgeon $(2 / 15)$ $(6,8)$ or the resident performing the placement of external ventricular drainage (1/15) (5). Two authors did not specify the participants in their checklists $(2 / 15)(7,9)$.

\section{Preventing incorrect site surgery}

The prevention of all types of wrong site surgery was almost ubiquitously one of the primary goals of the neurosurgical checklists $(11 / 15)(6-8,10-15,18,19)$. Three of the checklists $(3 / 15)(9,16,17)$ did not include this item because of their highly specific and narrow areas and sites of action, in which incorrect site surgery did not exist as an option. One report did not clarify this element (1/15) (5).

\section{Preventing complications due to incorrect positioning of the patients for a neurosurgical procedure}

Only 2 neurosurgical checklists $(2 / 15)(10,15)$ aimed to prevent complications due to incorrect positioning of the patients for a neurosurgical procedure. They were specially designed for the prevention of potential complications in an intraoperative MRI suite $(10,15)$. The sparse presence of this problem in the available neurosurgical checklists represents a noteworthy omission which must be corrected in their modifications or in the newly devised safety checklists.

\section{Preventing complications due to a failure of devices required for the neurosurgical procedure}

Only 5 out of 15 neurosurgical checklists (5/15) $(7,10,12,14,15)$ incorporated items targeting the prevention of complications due to a failure of the devices required for neurosurgical procedures. The safety check of all necessary systems and machines in the operating theatre, being closest to the prototype checks in aviation, is unduly underestimated in common safety efforts. This potential problem could lead to devastating consequences and must receive the deserved attention and exertions in the safety measures. 


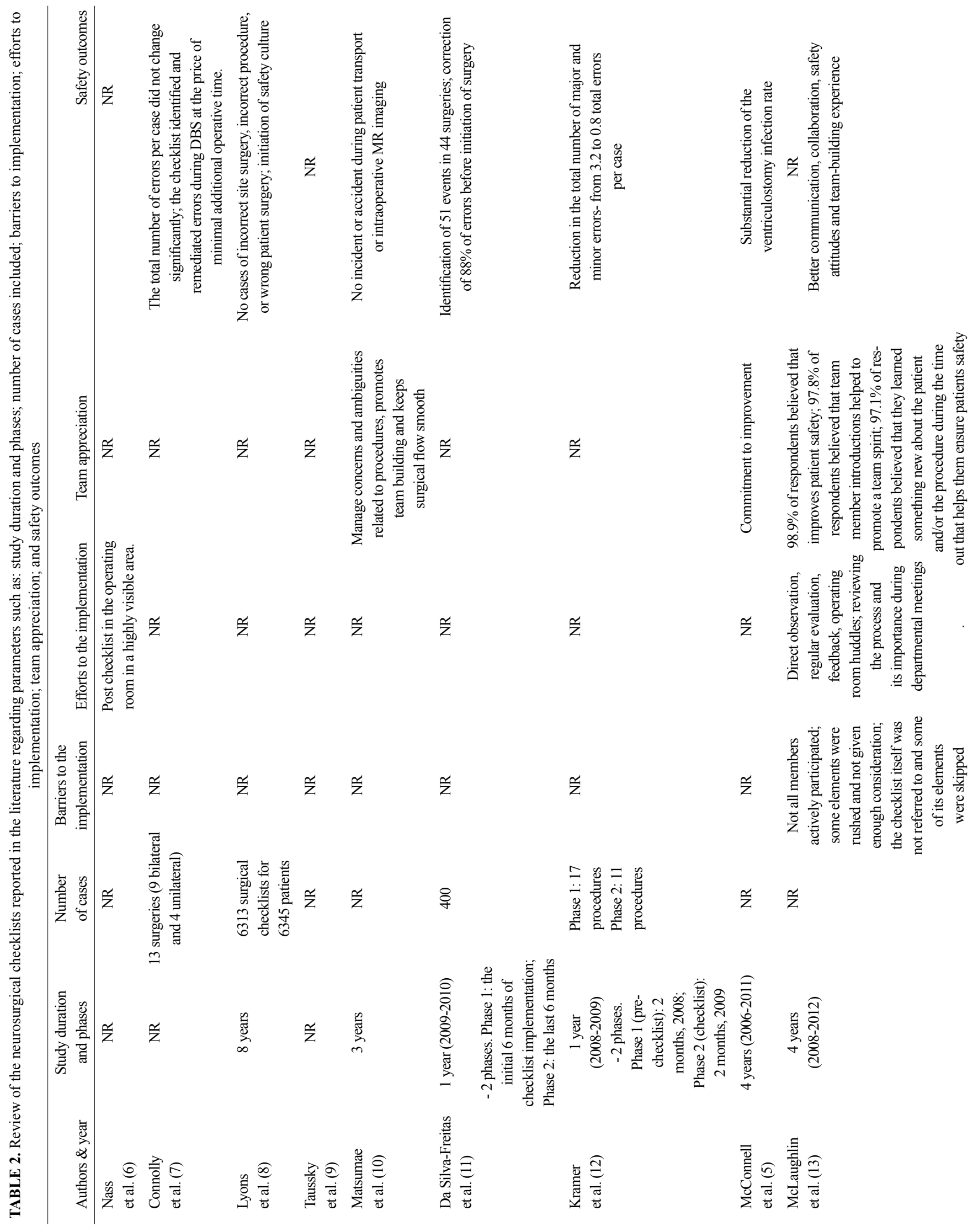

Balkan Med J, Vol. 32, No. 4, 2015 


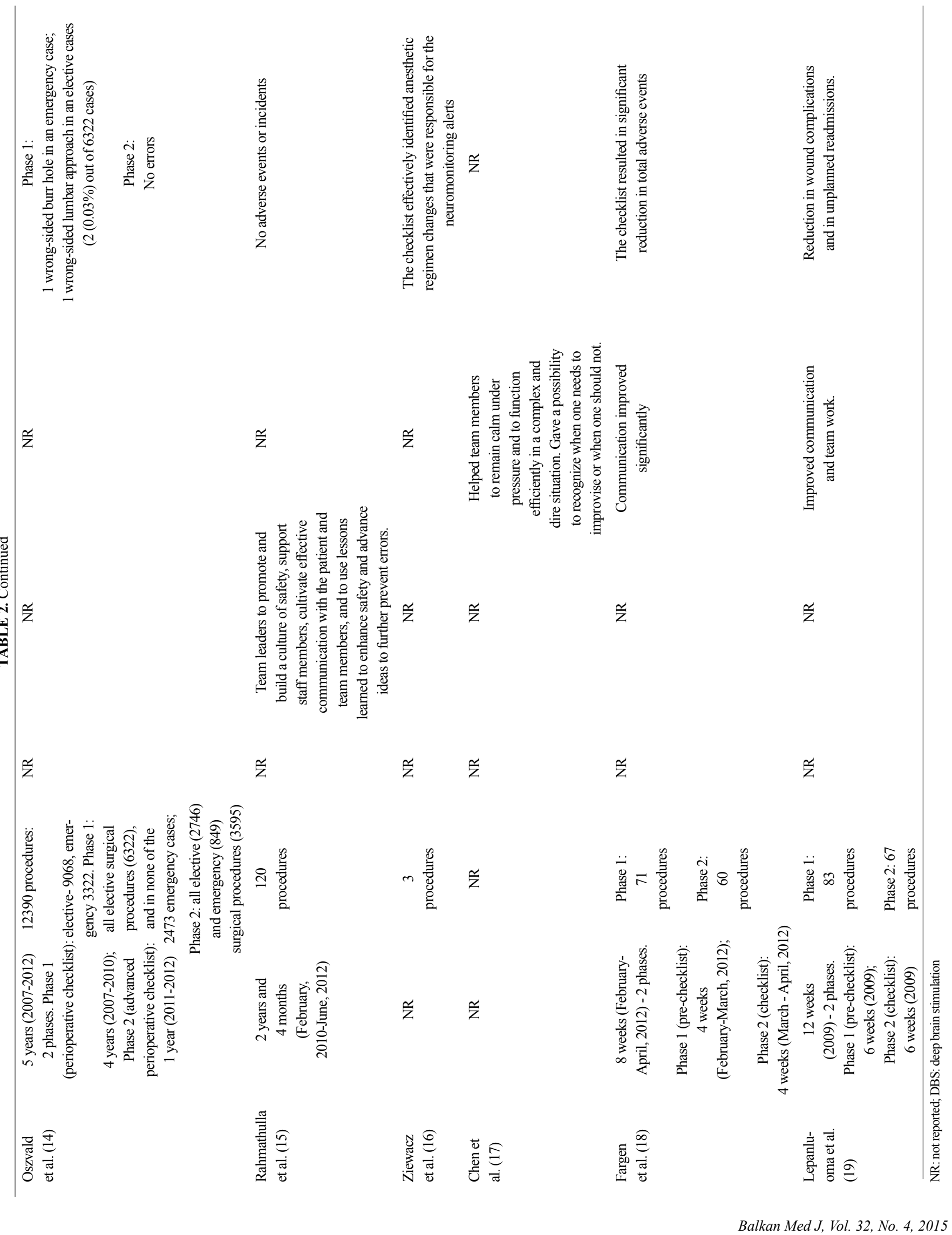




\section{Specific aims}

The specific aims of the available neurosurgical checklists varied according to their area of neurosurgical application (Table 1). It could not be expected that stereotactic checklists $(7,12)$ for example would resemble endovascular ones $(9,17,18)$. Naturally, they reflected the safety experience, attitudes and culture of the individuals and institutions responsible for their elaboration and implementation.

\section{Educational preparation and training regarding checklist implementation}

The implementation of the safety checklists as a rule is preceded by educational events and preparation. However, only 5 studies $(5 / 15)$ of the neurosurgical checklists reported educational efforts $(6,10,13,15,19)$. These measures included a checklist posted in the operating room in a highly visible area, educational training, lectures, videos, mock test and mock emergency code drill, and reading aloud a surgical safety manual and checklist during the period of preparation for intraoperative MRI.

\section{Time needed for checklist completion}

The time needed for checklist completion was detailed in only 3 series $(3 / 15)$ of neurosurgical checklists $(7,10,13)$. It ranged between 1 and 8 minutes. Not reporting the average time that the checklist application added to the total operative time could be considered a significant drawback of each of those papers. The regular time spent for the neurosurgical checklists in the operating theatre is very important in evaluating their cost-effectiveness.

\section{Study duration and phases}

Ten of the series (10/15) with neurosurgical checklists presented their study duration and phases. The most long-term study was that of Lyons et al. (8) which covered a period of 8 years and was not divided in phases. The series of Oszvald et al. (14) continued 5 years and included two phases (Phase 1, perioperative checklist, 4 years and Phase 2, advanced perioperative checklist, 1 year). McConnell et al. (5) and McLaughlin et al. (13) had 4 years of experience and no study phases. Matsumae et al. (10) and Rahmathulla et al. (15) reported 3 years and 2 years and 4 months, respectively, for their study duration and no study phases. Da Silva-Freitas et al. (11) presented their 1 year experience with a modified World Health Organization (WHO) neurosurgical checklist and compared the initial 6 months of checklist implementation with the following 6 months. Kramer et al. (12), Lepanluoma et al. (19) and Fargen et al. (18), in their studies lasting 1 year, 12 weeks and 8 weeks, respectively, compared equally prolonged prechecklist and post-checklist periods. In 5 papers $(6,7,9,16,17)$, the study terms were not discussed, nor were the eventual study phases.
The most reliable results could be achieved by series with more prolonged durations comparing pre-checklist and checklist phases. Hopefully, future studies in this field will cover these expectations.

\section{Number of cases}

The number of all reported cases secured by neurosurgical checklists to date is fewer than 20,000 (5-19). Oszvald et al. (14) described the biggest series which included 12,390 procedures controlled by a safety checklist. The next largest was the report of Lyons et al. (8), covering 6313 cases. The rest of the papers included 400 or less neurosurgical cases, with the smallest one being comprised of only 3 procedures (16) controlled by a safety checklist.

The comparison between a neurosurgical checklist applied in 3 cases (16) with another checklist used in over 12,000 procedures (14) would not be meaningful and reliable.

\section{Barriers to the checklist implementation}

Only one report (1/15) (13) presented the attendant barriers to the implementation of neurosurgical checklists. McLaughlin et al. (13) reported that not all members actively participated, some elements were rushed and not given sufficient consideration, the checklist itself was not referred to and some of its elements were skipped. The fact that the barriers to neurosurgical checklist implementation were rarely reported should not mislead the neurosurgical centers and colleagues who should consider starting to apply safety checklists routinely in their daily practice so that the process is smooth and uneventful.

\section{Efforts to the checklist implementation}

Three teams $(3 / 15)(6,13,15)$ described their efforts with the implementation of the neurosurgical checklists. NASS (6) posted the safety checklist in the operating room in a highly visible area. McLaughlin et al. (13) used direct observation, regular evaluation, feedback, and operating room huddles and reviewed the process and its importance during departmental meetings. Rahmathulla et al. (15) recommended team leaders promoting and building a culture of safety, supporting staff members, cultivating effective communication with the patient and team members, and using lessons learned to enhance safety and advance ideas to prevent further errors.

\section{Team appreciation}

The team appreciation of the neurosurgical checklist is of utmost importance for its successful application. This was reported in 6 out of 15 series $(5,10,13,17-19)$. Matsumae et al. (10) believed that the safety checklist managed the concerns and ambiguities related to the procedures, promoted team building 
and kept the surgical flow smooth. McConnell et al. (5) considered the checklist as a commitment to improved patient safety. According to McLaughlin et al. (13) the majority of the respondents in their study believed that the safety checklist improved patient safety, that team member introductions helped to promote a team spirit and that they learned something new about the patient and/or the procedure during the time, which helped them to ensure patient safety. Chen et al. (17) thought that the neurosurgical checklist helped team members to remain calm under pressure and to function efficiently in a complex and dire situation. It gave a possibility to recognize when one needed to improvise or when one should not. Fargen et al. (18) and Lepanluoma et al. (19) believed that the checklists significantly improved the team communication and work.

\section{Safety outcomes}

The question of the safety outcomes is the most important in the analysis of neurosurgical checklists.

In general neurosurgery, Lyons et al. (8) had no cases of incorrect site surgery, wrong procedure, or wrong patient surgery, which was regarded an initiation of safety culture. Da Silva-Freitas et al. (11) identified 51 events in 44 surgeries and achieved the correction of $88 \%$ of errors before the initiation of surgery. Oszvald et al. (14), in their series, had 1 wrong-sided burr hole in an emergency case and 1 wrong-sided lumbar approach in an elective case in their study phase $1(2(0.03 \%)$ errors out of 6322 cases) and no errors in phase 2. McLaughlin et al. (13) did not report any concrete safety outcome data but observed better communication, collaboration, safety attitudes and team-building experience. Lepanluoma et al. (19) achieved reduction in the wound complications and unplanned readmissions. Matsumae et al. (10) and Rahmathulla et al. (15) with their own specially designed checklists for neurosurgical procedures performed into an intraoperative MRI also reported no safety incidents or accidents.

In the area of stereotactic and functional neurosurgery, Connolly et al. (7) reported that the total number of errors per case did not change significantly. However, the checklist identified and remediated errors during DBS at the price of minimal additional operative time. Kramer et al. (12) identified a reduction in the total number of major and minor errors - from 3.2 to 0.8 total errors per case.

In vascular neurosurgery, the checklist of Fargen et al. (18) resulted in a significant reduction of total adverse events.

In spinal neurosurgery, Ziewacz et al. (16) with their checklist for intraoperative neuromonitoring alerts effectively identified anesthetic regimen changes that were responsible for the neuromonitoring warnings.

McConnell et al. (5), with the checklist developed by them for the placement of external ventricular drainage, achieved substantial reduction of the ventriculostomy infection rate.
In 3 papers (3/15), the safety outcome was not reported $(6,9,17)$.

\section{CONCLUSION}

To date, the world neurosurgical experience with checklists is quite limited compared to other areas of surgery and intensive care medicine. There are only a few neurosurgical centers with significant achievements in respect of duration of safety checklist application and number of secured procedures. However, the safety outcomes of the neurosurgical checklists, regarding the prevention of all types of wrong site surgery, complications due to the incorrect positioning of patients for a neurosurgical procedure, complications due to failure of the devices required for the specific intervention and some peculiar errors to a particular neurosurgical area or situation, are excellent and encouraging. The neurosurgical checklists represent an efficient, reliable, cost-effective and time-saving tool for increasing the patient safety and elevating the neurosurgeons' self-confidence. They are a step ahead in building a global safety culture in neurosurgery and as such they deserve active attention and the efforts of international and national neurosurgical societies.

\section{Ethics Committee Approval: N/A.}

\section{Informed Consent: N/A.}

Peer-review: Externally peer-reviewed.

Conflict of Interest: No conflict of interest was declared by the author.

Financial Disclosure: The author declared that this study has received no financial support.

\section{REFERENCES}

1. Grober ED, Bohnen JM. Defining medical error. Can J Surg 2005;48:39-44.

2. Devine J, Chutkan N, Norvell DC, Dettori JR. Avoiding wrong site surgery: a systematic review. Spine (Phila Pa 1976) 2010;35(Suppl 9):S28-36. [CrossRef]

3. Kamel I, Barnette R. Positioning patients for spine surgery: Avoiding uncommon position-related complications. World $J$ Orthop 2014;5:425-43. [CrossRef]

4. Cohen FL, Mendelsohn D, Bernstein M. Wrong-site craniotomy: analysis of 35 cases and systems for prevention. J Neurosurg 2010;113:461-73. [CrossRef]

5. McConnell DJ, Fargen KM, Mocco J. Surgical checklists: A detailed review of their emergence, development, and relevance to neurosurgical practice. Surg Neurol Int 2012;3:2. [CrossRef] 
6. North American Spine Society. Sign, mark and X-ray (SMaX): Prevent wrong-site surgery. Available at: https://wwwspineorg/Pages/ ResearchClinicalCare/PatientSafety/SignMarkXrayaspx. 2001.

7. Connolly PJ, Kilpatrick M, Jaggi JL, Church E, Baltuch GH. Feasibility of an operational standardized checklist for movement disorder surgery. A pilot study. Stereotact Funct Neurosurg 2009;87:94-100. [CrossRef]

8. Lyons MK. Eight-year experience with a neurosurgical checklist. Am J Med Qual 2010;25:285-8. [CrossRef]

9. Taussky P, Lanzino G, Cloft H, Kallmes D. A checklist in the event of aneurysm perforation during coiling. AJNR Am J Neuroradiol 2010;31:E59. [CrossRef]

10. Matsumae M, Nakajima Y, Morikawa E, Nishiyama J, Atsumi $\mathrm{H}$, Tominaga J, et al. Improving patient safety in the intra-operative MRI suite using an on-duty safety nurse, safety manual and checklist. Acta Neurochir Suppl 2011;109:219-22. [CrossRef]

11. Da Silva-Freitas R, Martin-Laez R, Madrazo-Leal CB, VillenaMartin M, Valduvieco-Juaristi I, Martinez-Agueros JA, et al. [Establishment of a modified surgical safety checklist for the neurosurgical patient: Initial experience in 400 cases]. Neurocirugia (Astur) 2012;23:60-9. [CrossRef]

12. Kramer DR, Halpern CH, Connolly PJ, Jaggi JL, Baltuch GH. Error reduction with routine checklist use during deep brain stimulation surgery. Stereotact Funct Neurosurg 2012;90:255-9. [CrossRef]
13. McLaughlin N, Winograd D, Chung HR, Van de Wiele B, Martin NA. University of California, Los Angeles, surgical time-out process: evolution, challenges, and future perspective. Neurosurg Focus 2012;33:E5. [CrossRef]

14. Oszvald A, Vatter H, Byhahn C, Seifert V, Guresir E. "Team time-out" and surgical safety-experiences in 12,390 neurosurgical patients. Neurosurg Focus 2012;33:E6. [CrossRef]

15. Rahmathulla G, Recinos PF, Traul DE, Avitsian R, Yunak M, Harper NT, et al. Surgical briefings, checklists, and the creation of an environment of safety in the neurosurgical intraoperative magnetic resonance imaging suite. Neurosurg Focus 2012;33:E12. [CrossRef]

16. Ziewacz JE, Berven SH, Mummaneni VP, Tu TH, Akinbo OC, Lyon R, et al. The design, development, and implementation of a checklist for intraoperative neuromonitoring changes. Neurosurg Focus 2012;33:E11. [CrossRef]

17. Chen M. A checklist for cerebral aneurysm embolization complications. J Neurointerv Surg 2013;5:20-7. [CrossRef]

18. Fargen KM, Velat GJ, Lawson MF, Firment CS, Mocco J, Hoh BL. Enhanced staff communication and reduced near-miss errors with a neurointerventional procedural checklist. J Neurointerv Surg 2013;5:497-500. [CrossRef]

19. Lepanluoma M, Takala R, Kotkansalo A, Rahi M, Ikonen TS. Surgical safety checklist is associated with improved operating room safety culture, reduced wound complications, and unplanned readmissions in a pilot study in neurosurgery. Scand $J$ Surg 2014;103:66-72. [CrossRef] 